\title{
Moaning and Eye Contact: Men's Use of Ambiguous Signals in Attributions of Consent to their Sexual Partners
}

\begin{abstract}
In recent years, there has been increasing pressure on men to take a proactive role in preventing sexual violence. On college campuses, this pressure has been formalized into affirmative consent policies that require all students to actively seek consent from their sexual partners through unambiguous verbal or physical signals. This study uses data from 25 semi-structured interviews to explore how undergraduate men make sense of sexual consent after cultural and organizational pressure to be more proactive in preventing sexual assault. Participants answered questions about their recent sexual experiences and their attitudes toward campus sexual consent policies. Findings indicate that while participants understand and condone key elements of sexual consent, they do not consistently apply reliable strategies to ensure that their sexual interactions are consensual. Instead, they use ambiguous social cues that are common in both consensual and nonconsensual sexual interactions, which reinforce the cultural notion that consent is unclear.
\end{abstract}

Keywords: sexual assault, masculinity, consent, gender, ambiguity 


\section{Introduction}

Sexual violence is widespread in the United States. In recent years, college students' experiences of sexual assault have received particular attention. Recent research estimates that between $19 \%$ and $23 \%$ of undergraduate women experience either an attempted or completed sexual assault during college (Cantor et al. 2015; Krebs et al. 2007) and as many as 11\% of undergraduate men commit a rape before graduation (Swartout et al. 2015). To address the high rates of violence, college campuses have done something few other organizations have- they have formally instituted prevention measures, many of which explicitly require men to take a proactive approach to sexual assault prevention. Among the most widespread changes is a movement to define campus sexual assault through affirmative consent policies and trainings, which require all students — including and perhaps particularly men — to actively seek consent from their sexual partners through clear verbal or physical cues as a way to minimize miscommunication.

The theory that sexual assault on campus is simply miscommunication between sexually inexperienced young adults has been prevalent in the popular media and explored in depth by scholars across disciplines. While college students' negotiations of consent may appear clumsy or confused (Hirsch and Khan 2020), the sexual scripts employed become clearer through the use of a gendered lens (Muehlenhard et al. 2016). Despite evidence that men and women use different cues to convey sexual desire (Beres 2010; Hickman and Muehlenhard 1999; Jozkowski et al. 2014), academics have long refuted the claim that young men cannot understand women's sexual signals (Hickman and Muehlenhard 1999; Kitzinger and Frith 1999). Instead, many have suggested that men's alleged misinterpretation of women's sexual signals is better described as the systematic marginalization of women's voices (Abbey 1982; Abbey 1987; Osman 2003) that 
can be exacerbated in specific social environments, particularly those common to college campuses (Armstrong, Hamilton \& Sweeney 2008; Martin 2016; Hirsch et al. 2019; Wade 2017). Researchers have found that men who engage in hostile masculinity are especially likely to claim to misinterpret a partner's sexual cues (Jacques-Tiura et al. 2007) and reinterpretation of a victim's mundane actions as indicators of consent is a common tactic used by incarcerated rapists to justify their violence (Scully \& Marolla 1983). In sum, these findings suggest that traditional consent acquisition practices are embedded in a gendered hierarchy that prioritizes men's sexual interests and allows men to dictate what is and is not consent. The popular notion that most campus sexual violence is mere miscommunication reinforces and naturalizes these gendered hierarchies surrounding consent-seeking practices.

Ideally, campus affirmative consent policies and the associated trainings would challenge these gender hierarchies and put an end to the myth that sexual violence is the result of miscommunication over ambiguity. To put it simply, those accused of sexual assault could no longer revert to an excuse that they could not tell that they had traumatized a sexual partnerthey would be expected to take a more proactive approach after university interventions. However, there are many reasons to anticipate that affirmative consent programming would fall short of these goals, particularly since men are commonly viewed—and accepted—as resistant to sexual assault prevention efforts and ambiguity in sexual scripts offers advantages to men in heterosexual sexual pairings they may be reluctant to give up voluntarily. The current study seeks to understand how men have responded to increased organizational and cultural pressures to take a more proactive response in sexual violence prevention through seeking clear affirmative consent in their sexual encounters. In particular, the current study explores whether heterosexual 
men self-report changing their behaviors in a way that relinquishes control over consent narratives and the associated gender hierarchies.

Redefining sexual consent. Over the past decade, the definition of consent has evolved on college campuses. Previously, a victim would need to offer evidence that sexual consent had been absent or explicitly revoked to categorize a sexual encounter as assault. Activists and educators criticized these policies for placing an undue burden on victims, leading to low reporting rates, victim blaming, and retraumatization in cases where revocation of consent was not demonstrable (e.g., Friedman and Valenti 2008). Instead, reformers recommended affirmative consent policies. While affirmative consent policies vary between institutions, they generally include three main criteria: (1) consent must be given knowingly; (2) consent must be given voluntarily; (3) consent must be given affirmatively and unambiguously (as early statewide examples, California Legislature 2014; New York Senate 2015). Notably, affirmative consent policies typically privilege verbal methods of obtaining consent, but most also permit enthusiastic participation in sexual action as acceptable evidence of a partner's consent, so long as that enthusiastic participation is ongoing throughout the entirety of a sexual encounter. Additionally, many collegiate policies elaborate on scenarios in which consent cannot be given (e.g., while incapacitated or under duress) and scenarios that are commonly mistaken as consensual (e.g., lack of protest or a pre-established sexual relationship).

Affirmative consent policies have become common college campuses with $80 \%$ of colleges defining sexual assault using an affirmative consent standard (Armstrong et al. 2019). At the time of data collection for this study, two states had passed legislation that linked state colleges' adoption of affirmative consent policies to their eligibility for state funding (California 
Legislature 2014; New York State Senate 2015). More commonly, colleges voluntarily implemented affirmative consent policies drafted by administrators, as is the case with the university attended by men in this study. On any campus that has adopted an affirmative consent policy, obtaining sexual consent is not a recommended practice, but mandatory for all students.

Men, consent, and ambiguity. Regardless of campus policy, men's willingness to adopt an affirmative consent approach in their own sexual experiences is not guaranteed and subject to many cultural forces. Research on men's engagement in anti-violence and other feminist activist efforts is divided. Even in evaluating similar programs, researchers have come to disparate conclusions about men's willingness to engage in sexual assault prevention (Piccigallo et al. 2012; Rich et al. 2010), perhaps related to the heterogeneity expected in men's responses to such programs (Malamuth et al. 2018). As sexual violence becomes a more widely discussed topic on college campuses, it is possible that men will use anti-violence rhetoric as a way to assert their masculinity and sexual prowess without changing their behavior (Pascoe and Hollander 2016) and even men who actively participate in prevention programming may get the impression that their own behavior should be exempt from scrutiny (Messner 2016) or might unintentionally reify gendered hierarchies that allow violence to occur (Bridges 2010; Bridges and Pascoe 2014; Connell and Messerschmidt 2005; Masters 2010). For these reasons, men's successful engagement with affirmative consent programming might be more complicated than their stated desires to prevent sexual assault and pursue purely consensual sex.

In particular, sexual ambiguity offers all men benefits in traditional heterosexual sexual parings. Ambiguity in narratives of sexual encounters allows men to exaggerate their sexual prowess in verbal performances of masculinity (Currier 2013) and receive more sexual pleasure 
than their partners during casual sex (Armstrong, England, and Fogarty 2012). Even though these advantages do not come from participating in sexual violence, the ambiguity they require and their contributions to a larger sexual script cannot be separated from violence (Simon and Gagnon 1986). A sense of ambiguity makes bystanders less likely to intervene in a sexual assault they witness (Pugh, Ningard, Vander Ven and Butler 2016) and leads many to discredit survivors' claims of victimization (as an example, Stepp 2007) or engage in victim blaming (Frese, Moya, Megias 2004; Randall 2010; Ryan 1976). Ambiguity can also make it more difficult for survivors themselves to categorize what happened them (Peterson and Muehlenhard 2004; Smith and Martinez 1996; Warshaw 1988), effectively blocking victims from reporting or accessing resources (Hlavka 2014; Holland and Cortina 2017). Perhaps most importantly, ambiguity permits subjective interpretation of the presence or absence of consent, which researchers have suggested leads men to label ambiguous events as nonconsensual at a drastically lower rate than women (King et al. 2020). In short, the normalization of ambiguity in sexual interactions and narratives about them — whether consensual or not—can make the tasks of defining and responding to violence harder.

\section{Data and Methods}

Participants. The data for this study comes from 25 semi-structured interviews conducted during 2015 with undergraduate men at a large university in the mid-Atlantic region. The university they attend had adopted an affirmative consent policy and instilled mandatory trainings for students about sexual consent, including a sexual assault awareness seminar at first year student orientation and an online course about sexual violence that students of all class standings must complete every semester. Students may also engage in additional education about sexual consent 
through engagement with student clubs, fraternities, or certain classes available on campus. The university's sexual violence prevention training has received national recognition for its rigor. In comparison to men in the general public, the men in this study have received an exceptionally high amount of formalized training on the topics of sexual violence and consent-seeking. While this study does not specifically evaluate any program on campus, the data collection site was selected based on the many opportunities participants would have had to receive both formal and informal education about approaches to preventing sexual violence in their sexual encounters.

I recruited heterosexual men of traditional college age who described themselves as sexually active from six lower division courses ${ }^{1}$ with at least 50 students. One course from the most popular discipline in each college at the university was selected to ensure more ideological diversity among participants than typical in convenient samples focused in a single discipline. Students enrolled in these classes were invited in person ${ }^{2}$ by a mixed-sex pair to participate in a study about college men's sexual behavior and were informed that they would receive a $\$ 10$ gift card for their participation.

Although the sampling method was not random, the participants make up a representative sample of their university. Participants' ages ranged between 18 and 24 with a mean age of 20. Of the participants, seventeen identified as White (68\%), three identified as Black (12\%), two identified as Asian American (8\%), two identified as non-white Latino (8\%), and one identified as biracial (4\%). Participants reported a variety of religious backgrounds including Catholic (52\%), Jewish (20\%), Protestant (12\%), Muslim (4\%), and Latter-day Saints (4\%). Participants belonged to various class standings, including first years $(20 \%)$, second years $(48 \%)$, third years

\footnotetext{
- The disciplines used for recruitment were animal studies, economics, accounting, computer science, mechanical engineering, communications, and journalism.

2 There was one exception in this recruitment protocol. One college at the university had a policy prohibiting visitors outside of the discipline. Students from that college were recruited via email with assistance of the professor.
} 
$(12 \%)$, fourth years (16\%), and students who had spent at least five years enrolled in the university (4\%). Of the participants, four belonged to a fraternity (16\%). None of the participants currently represented the university on an official athletic team; however, one participant had previously competed on an athletic team at another university and nine participants (36\%) participated in intramural athletic organizations.

Methods. I used a semi-structured interview approach to explore the participants' experiences with sex in long-term relationships and hookups. I also asked participants about sexual experiences that they regretted. To avoid socially desirable responses, I did not use the word "consent" during interviews sections in which participants described their recent sexual encounters or regretted sexual events. Instead, I used multiple lines of questioning about how participants could tell their partners wanted to engage in sexual activity with them at various points during each interactions and specifically probed for any verbal cues that helped them gauge a partner's willingness to engage in sexual activity. I only used the word "consent" in a final cluster of questions about the participants' attitudes toward affirmative consent policies and their willingness to seek consent according to affirmative consent requirements.

To guide the portions on their reported sexual behaviors, I asked participants about their most recent sexual encounter in a long-term relationship and in a hookup, although many participants also told me about earlier experiences that informed their attitudes toward their most recent encounter. Drawing on the methods of Armstrong and colleagues (2012), I let the participants use their own definitions of relationship and hookup, but their applications appeared similar. Participants categorized a sexual experience as belonging to a long-term relationship if they had exclusively dated that partner for an extended period of time or had engaged in a 
conversation defining their relationship. When asked about hookups, participants typically mentioned that they had participated in hookups that did not involve sex, but most participants chose to talk about their most recent hookup that ended in intercourse. Some participants had not engaged in hookups that included intercourse, but instead told me about when they had engaged in kissing or other sexual acts that they believed could have led to intercourse. Interviews lasted between 24 and 76 minutes with a median length of 48 minutes. ${ }^{3}$

All interviews used for this study were conducted by a woman. To understand the effect of my gender on my participants, I employed a male undergraduate research assistant to conduct supplementary interviews. The research assistant was trained over the course of three months, including conducting mock interviews. I reviewed recordings of his interviews regularly to ensure that his demeanor and interview style matched my own. In general, the substance of the participants' comments was similar between the two sets of interviews; however, the participants were more reluctant to give detailed accounts of their past sexual encounters when discussing them with another man. This was particularly true for the sections of the interview on sexual encounters occurring in the context of a romantic relationship, perhaps indicating their unfamiliarity or discomfort with discussing issues of emotional intimacy with other men. Accordingly, interviews conducted by a man were significantly shorter and the data are less detailed, producing thin description. They are not included in the data for this study. In contrast, many of the participants working with a female interviewer commented about how they felt unencumbered during their interviews, especially when discussing their feelings or sexual insecurities. Many participants commented that they felt comfortable sharing information about their sex lives that they had never told anyone before. Only one participant expressed discomfort

\footnotetext{
- The variation in interview length can be attributed to the number of sexual experiences a participant had to share. Participants who had not engaged in a hookup had shorter interviews.
} 
after an interview with a woman — he felt embarrassed by how much he divulged about his feelings toward his ex-girlfriend — but did not wish to withdraw his interview from the data, nor was that portion of his interview relevant to the analysis presented in this paper.

During my analysis, I used NVivo qualitative analysis software to code for the cues participants reported to use to attribute consent (or willingness to engage in sexual interaction) to their partners. I separated these codes into two separate categories based on which portion of the interview they were mentioned by a participant (i.e., reflection on their previous behaviors or in their ideal strategies for seeking consent) and used open-coding to categorize the cues (e.g., verbal, physical, or other; ambiguous or unambiguous). Since there is no widely accepted scholarly definition of ambiguity in sexual interactions, I defined ambiguity as the absence of the three primary pillars of consent as found in state laws impacting collegiate policies: (1) consent must be given knowingly; (2) consent must be given voluntarily; (3) consent must be given affirmatively (California Legislature 2014; New York Senate 2015). For example, a signal was interpreted ambiguous if it could be seen as sexual unknowingly (e.g., not wearing a bra in public, drinking alcohol) or involuntarily (e.g., increased heart rate, increased breath rate). For a signal to be coded as unambiguous, it had to meet all three criteria (e.g., reciprocated kissing, initiating a sexual act). I analyzed the participants' reported attitudes toward affirmative consent policies and practices, which I compared to an affirmative consent standard using the same methods. To deepen analysis, I separated the data based on the context of the sexual encounter described by a participant (i.e., hookup or relationship sexual events) and based on the ages and class standing of the participant, but found no meaningful differences between the two categories that were relevant to this paper. I use pseudonyms to maintain the confidentiality of participants and omitted any potentially identifying information in the analysis. I made small edits for clarity. 
In the sections to follow, I describe the participants' attitudes toward affirmative consent policies and efficacy in defining consent and producing ideal strategies for unambiguous consent seeking. Afterward, I compare their idealized beliefs about consent-seeking to their reported behaviors. To ease comparison for the reader, I will begin each section with the frequencies at which each code was applied; however, the analyses that follow will be focused on the qualitative differences between participants' idealized beliefs and self-reported behaviors.

\section{Findings}

Defining Consent. In general, the participants in this study endorsed affirmative consent as an ideology and they demonstrated relative ease in producing a definition of consensual sexual behavior. Of the participants who struggled to define consent, they seemed to trip over their words, but ultimately understand its key principles - that consent must be knowing, voluntary, and affirmative. As an example, Ethan, a white 20-year-old, paused and interrupted himself, but ultimately defined consent with:

Um... If I had to define consent, um, I would say to give consent would be to, um, say that you're able and willing to, um, engage in whatever they're proposing, um, and that they have your permission to engage in it. If that makes sense.

Most commonly, the participants defined consent accurately and concisely. Similar to the findings from previous studies (Jozkowski et al. 2014), the participants in this study typically defined consent as either an explicit agreement to have sex or as sex wanted by all parties. In the former group, Elliot, a white 20-year-old, emphasized the importance of communication when he defined consent as, "[A] verbal or nonverbal cue that the other person wants to continue this particular act or initiate this particular act." In the latter group, Luis, a Latino 18-year-old, had a 
simpler interpretation of consent based in desire. He said, "The fact that both sides are willing to do the same stuff." Participants like Elliot offered definitions of consent that had a clear course of action for implementation (e.g., looking to verbal or nonverbal cues); however, even desirebased approaches like Luis' reflect an understanding of affirmative consent.

Few participants rejected or contested affirmative consent as a whole. Typically, participants who questioned affirmative consent did so under the assumption that affirmative consent required an explicit verbal discussion about sexual intentions - an assumption not reflected in any state and few campus policies (Armstrong et al. 2019). As an example, Will, a white 19-year-old, defined consent as:

Consent on both sides is-now, if I say it's a verbal agreement, then I would have by my definition violated my own definition of sexual consent, so I'm not going to say verbal. I'm gonna say it's common sense. Common sense, but it's hard to articulate. It is when both partners—or multiple partners—have mutually agreed either by physical or verbal indications that they are willing to participate in sexual intercourse.

Ultimately, Will embraces the ideology of affirmative consent, calling it "common sense" and clearly defining consensual sex as a mutual willingness to participate in sexual events. Only a single participant, Michael, a white 20-year-old, clearly resented affirmative consent, wanting instead a return to a sexual system in which "if a girl does not want to do something, then she'll let you know [emphasis added]." One additional participant, Ray, a black 24-year-old, refused to define consent, but insisted, "It's important that you try to do as much [as you can] to ensure that it's consent," and provided multiple example of situations in which he had ended a flirtation or sexual interaction because he believed he did not have clear consent, adding: 
If you're unsure and you're trying to figure [it] out, I would ask her. Ask. 'Cause sometimes it's like she ripped her clothes off or whatever. That could be consent, but at the same time, a girl could rip her clothes off and then hop in the shower and it's like, “No, I didn't want to have sex with you. I wanted to hop in the shower. "... It's nothing that I personally think can [have] a definition for it. It's something you have to keep in mind at all times.

Despite an unwillingness to define consent, Ray was among the most vocal advocates for consent and provided one of the most comprehensive descriptions of the complexity and potential ambiguity in consent-seeking. In general, even participants who put forth critiques still endorsed the spirit of affirmative consent.

As a group, the participants demonstrated that they understood the core tenets of affirmative consent policies and actively endorsed affirmative consent as an idea.

Consent-Seeking Strategies. The participants were equally as enthusiastic in their description of tactics to seek consent from their partners. All but one insisted that they applied what they had learned on campus about affirmative consent to their sexual encounters. Of the strategies mentioned by participants when speaking broadly about their consent-seeking practices, $34 \%$ referred to verbal signals that were unambiguous and explicitly sexual (e.g., asking for consent), $22 \%$ referred to physical signals that explicitly sexual (e.g., enthusiastic participation in kissing), $19 \%$ referred to signals that hinted to a partner's state of mind (e.g., knowing a partner was comfortable), and $16 \%$ relied on the absence of a signal that a partner had revoked consent (e.g., not asking to stop). Many participants claimed to use multiple signals to determine whether or not their partner(s) had consented to a sexual action, such as enthusiastic participation in physical 
sexual activity as well as the absence of signals that consent had been revoked, which, together, strengthened their certainty that they had their partner(s)' consent.

Most commonly, participants insisted that they explicitly asked for affirmative consent. Most participants assured me that they explicitly verbally communicated with their partners about their sexual intentions - and most said they did so every time they had sex. Ethan, a white 20-year-old, provides a clear example. He explained, "My personal favorite [way to get consent] is to straight up just ask them. Because I feel like that's the quickest, easiest, and with the least amount of gray area." Later he added, "I always ask for consent." Will, a white 19-year-old, similarly mused, "I’ve never been in a hookup where I haven’t asked [for consent]."

Participants also commonly cited looking to a partner's physical cues as a way to ensure they had their partners' consent, referring to a partner's enthusiastic participation in a sexual act. As an example, Josh, a white-23-year old, described, "[I make sure] they're always giving something back. Like a confirmation in some sense. Like if you're making out with them, they're not struggling to push you away. They're kissing you back." To Josh, evidence of consent came from the combination of a partner's enthusiastic participation with a lack of evidence that consent had been withdrawn. Other participants took more conservative stances, suggesting that the best strategy to seek consent non-verbally is to allow a partner to initiate the sex acts they want. As his primary strategy, Tal, an Asian-American 19-year-old, explained, "When I'm in situations where I do end up hooking up with someone, they're usually the one to initiate it with me, you know? And because of that, I'm pretty sure they consent to it." In each type of strategy based on physical signals, participants captured the requirement for enthusiasm and agency in a partner's behavior. The strategies relying on verbal and physical signals of consent met the standards set by affirmative consent policies. 
Even though nearly all participants condoned affirmative consent in theory, there was a minority whose ideal strategies for consent-seeking would not meet an affirmative consent standard. Instead, they made vague comments about a partner's state of mind or a lack of indicators that consent was absent. There were very few participants who exclusively mentioned these types of strategies. Only four participants provided only indicators of an absence of consent as their ideal strategies for consent-seeking. For example, Michael, the sole participant who had disparaged the affirmative consent standard, said, "I just always try and, you know, make sure my partner is comfortable with what we're doing and the second I hear, 'I'm not comfortable with this,' or, 'This isn't doing it for me,' I'll stop.” Similarly, Zachary, a biracial 22-year-old, articulated, “I expect [my partners] to say no when they don't want to. And yeah, the absence of the no confirms [that I had consent]." These participants made up a small minority who did not produce at least one strategy of consent-seeking that met an affirmative consent standard, but an important one. They most clearly represent a group of men who might condone affirmative consent as a theory, but still place the burden of sexual assault prevention on women.

Whatever their methods to gauge consent, the participants insisted that they used them reliably. In response to the question, "Have all of your sexual experiences been consensual?" only two answered no. It was common for participants to reference consistent use of the strategies they mentioned as evidence that they had never sexually assaulted anyone.

Consent-Seeking in Previous Encounters. While the participants as a group condoned affirmative consent policies and claimed to consistently apply clear strategies for consent-seeking, their descriptions of gauging a partner's willingness to engage in their recent sexual encounters relied primarily on ambiguous cues that would not meet an affirmative consent standard. Of the signals 
referenced by participants describing their most recent sexual encounters, $39 \%$ referred to ambiguous and non-sexual physical signals (e.g., eye contact, dancing together), $20 \%$ referred to physical signals that were explicitly sexual and varied in degree of ambiguity (e.g., kissing), 13\% referred to explicit verbal conversations about sex (e.g., asking for consent), $8 \%$ referred to verbal conversations not on the topic of sex (e.g., invitations to cook together), $4 \%$ referred an absence of signals of revoked consent (e.g., telling a participant to stop), and 3\% referred to signals that hinted at a partner's state of mind (e.g., being comfortable). Figure 1 visualizes the participants' ideal strategies to seek consent in comparison to their reports of consent-seeking behaviors in their previous sexual encounters (see Appendix A).

The starkest disparity between the participants' ideal and actual consent-seeking behaviors came from their reports of engaging in explicit conversations about consent. While explicit conversations about sexual expectations comprised a third of the ideal strategies participants claimed to use, they made up only $13 \%$ of the signals they recalled drawing upon in their recent sexual encounters. When an explicit dialogue about consent did occur, a woman usually initiated it. In $75 \%$ of the cases in this dataset, it was the participant's partner who asked for consent. When a man initiated a conversation about sexual expectations, he usually did not ask for consent, but instead spoke as if his partner's consent was implied. As an example, Ethan, a white 20-year-old, described the beginning of his most recent hookup: "I was like, 'Hey, we don't have to do anything if you don't want to. Or if we start and you change your mind, we can stop.' And she was like, 'Okay." By telling his partner that she could revoke consent at any time, Ethan recreated a traditional framework in which his partner became responsible for preventing an act of sexual violence during their encounter. Ethan did not continue to verbally check in with her, but instead expected she would speak up if she felt violated by him. 
Even in the exceptional cases in which a participant initiated a discussion of consent in the form of a question, he would quickly add that he did not seek consent in this form each time he had sex. For example, Ray, a black 24-year-old, described how he and his girlfriend decide whether or not to have sex:

So if we're chillin', she could be like, "Want to have sex?" And I'll be like, "Yeah, let's have sex." Or I'll be like, "Want to have sex?" And I'll be like, "Yeah, let's have sex. "[But] that's not all the time or whatever.

Participants like Ray used explicit verbal communication about sexual expectations as one way to seek consent, but they did so much less consistently than they had claimed they would when speaking about their ideal strategies.

Instead of consent-seeking through explicit verbal signals, participants most commonly reported relying on their partners' physical cues, many of which were ambiguous and often nonsexual in nature. These ambiguous cues were actions that could reasonably take place between two people who have no romantic or sexual relationship (e.g., smiling at each other) or could reasonably affect an individual in a nonsexual scenario (e.g., elevated heart rate), making them inherently ambiguous. Accordingly, these cues did not clearly meet the standard of enthusiastic participation required by an affirmative consent framework of being knowing, voluntary, and affirmative indicators of interest in sexual activity. Table 1 lists the most common ambiguous physical and nonsexual signals invoked by participants.

Ambiguous physical and non-sexual signals made up 39\% of the cues participants reported using to attribute consent to their partners. As a group, these cues were numerous and varied greatly, making it especially unlikely that women would be aware that their actions could be - and, in these scenarios, were - interpreted as indicators of consent. Out of 224 physical and 
non-sexual cues mentioned, most were only referenced once or twice by a single participant. As an example, Jaylen, a black 18-year-old, used a string of his partner's physical nonsexual actions to explain how he became certain that sex would occur between them:

She knew what she was doing. Everything she did screamed sex... She wore no bra. She clearly knew what she was doing. She put on some great music. I love music, so she knew every right thing to do... [Before that day, we had gone to] this party and we were riding back. We had a small little car with seven people and she chose to sit on my lap... We all get home together and she chilled with me the whole night... [The day we had sex, she was] twerking on my bed, laying down, stretching. Oh my gosh. She's a dancer, so she really got on my bed, hit a split, and said, "Oh my god. I'm so sore."

It is entirely possible - and even probable - that Jaylen's partner consented to have sex with him, but the indicators he invoked in describing the encounter as consensual are not reliable evidence of that. Jaylen's partner might have stretched before him as a way to entice him, but she might have simply needed to stretch after dance practice. She probably passed many men on her way to meet Jaylen at his dorm, but her decision not to wear a bra was certainly not a sexual invitation to each of them. Similar to Jaylen, other participants invoked signals like receiving a specific emoji in a text message or a woman brushing up against them at a party as signs of consent. The participants sexualized these otherwise mundane interactions to craft their narratives of consent to ultimately suggest that any woman who engages in polite discourse with them might have consented to engage in sex or could be blamed for sending mixed signals.

As evidence of the problematic nature of these ambiguous signals, consider the way participants in this study thought about moaning. Out of the ambiguous physical nonsexual cues, moaning was by far the most commonly cited by participants as a signal of consent $(n=25)$. Once 
sexual contact had begun, many participants relied solely on a combination of moaning and involuntary physiological actions as indicators of ongoing consent. When operating from a presumption of pleasure, the participants' narratives appear consensual and mutually enjoyable. However, the same excerpts also would not be unusual in a description of sexual violence as indications of pain. For example, Josh, a white 23-year-old, described his partner's behavior during a recent sexual event with, "I always think moaning is a good [sign]. [And] increased heartrate and then obviously when women get more wet." Moaning can be a signal of discomfort and increased heartrate and vaginal lubrication can be a response to trauma (Levin and Berlo 2004). Similarly, Alex, a white 20 -year-old, gave a similarly ambiguous description of his most recent hookup when he said, "She was moaning a lot and her eyes were closed and she gave me-I had a lot of scratches on my back." Scratching, too, can be a signal of pleasure or pain. As with Jaylen, it is entirely likely that Josh and Alex's experiences were entirely consensual, but it is concerning that the narratives of consent are similar to narratives of sexual abuse.

As the most mundane example of ambiguous narratives, participants commonly brought up eye contact as an indicator of consent $(n=11)$. When pressed for further details, most participants struggled to explain what differentiated ordinary eye contact — like the eye contact we made during our interviews - from sexualized eye contact that indicated consent. Many made no such distinction at all. Ray, a black 24-year-old, gave the most attention to the subject. After giving "eyes" as his one-word answer about how he knew his most recent hookup would take place, I asked him to elaborate. He went on, “It's like an interest — a curiosity. It's like when you look at someone with a curiosity to know more about them... I can see them like, 'Hmmm. What is that guy like in bed?'" I asked Ray to physically demonstrate what this type of eye contact would look like. He narrowed his eyes slightly. The difference was nearly imperceptible to me 
even when I explicitly looked for it. He explained simply, “It's subtle.” Few participants used eye contact as a sole indicator of consent the way that Ray did, but even those who used many different cues still relied exclusively on mundane and ambiguous signals, such as laughing at a joke, listening carefully, or not looking for an excuse to leave a conversation. None of these signals meet the standards of affirmative consent that the participants endorsed.

Even when participants' use of physical signals referred to explicitly sexual interactionwhich made up $20 \%$ of the indicators of consent mentioned - the cues were often still ambiguous or applied liberally by the participants. Most often, kissing or the removal of clothing indicated to the participants that they had consent for most - if not all—sexual actions, including vaginal intercourse. As with the participants who used verbal cues, they believed that a partner's participation in any explicitly sexual interaction promised consent for the duration of the sexual encounter. They did not report seeking ongoing indicators of consent. For example, Josh, a white 23-year-old, said that he "knew" his girlfriend was willing to have sex with him when she "took off her clothes and jumped on top of me and started making out with me." He did not describe seeking any additional indicators of consent later on. Andrew, an Asian-American 19-year-old agreed. He believed he had consent to vaginal sex during his most recent hookup when "clothes started coming off." As with other ambiguous physical signals, the removal of clothing in front of another person is often correlated with an interest in sex, but many sexual assaults also take place following the voluntary removal of clothes. Some participants who mentioned kissing or removal of clothing as an indicator of consent also commented on enthusiastic participation in other sexual events (e.g., asking for a condom, engaging in oral sex), but this was rare. 
Ultimately, only one participant described using physical signals as indicators of consent in the same way in describing ideal tactics for seeking consent and in reflection on a recent sexual encounter. Ethan, a white 20-year-old, described:

It wasn't me doing everything. She kind of took turns where I'd be in control and then she'd be in control. [At one point] I was on top and then she... flipped me over so I was on my back and then [she] got on top... I assume if she had felt uncomfortable or wanted to stop, when I backed off to allow her to take over, she wouldn't have.

Ethan's narrative of his most recent hookup included a key element of affirmative consent that the other men's did not: he described allowing his partner to share control throughout the entirety of their encounter. It is this element that makes his partner's consent clear in his narrative.

Overall, the participants' narratives of consent-seeking and attribution in their recent sexual experiences were quite different from the ideal strategies they shared when speaking generally about their beliefs about affirmative consent. The participants did not engage in explicit verbal conversations about consent or sexual expectations as often as they asserted they would. When those conversations did take place, they were usually initiated by their female partners or less open-ended or consistent than described in the abstract. The participants also relied on different types of physical cues that were more ambiguous than those they claimed they regularly used, seeing consent in scenarios that could be considered mundane by others.

\section{Discussion}

This study explores the way college men conceptualize and apply notions of affirmative consent in the wake of changing policies addressing sexual assault prevention on campus_-and broader attitudes about men's role in addressing sexual violence across society. Overall, the men 
in this study endorsed affirmative consent, could clearly define the concept, and claimed to regularly apply specific and unambiguous strategies to ensure their sexual encounters were consensual; however, their narratives about consent attribution in their most recent sexual encounters did not reflect the ideologies they claimed to hold. While participants claimed to primarily obtain consent through explicit verbal communication about sexual expectations or physical cues akin to enthusiastic participation in sexual activity when asked about "affirmative consent," the participants' reflections on their past sexual experiences suggested that they more often attributed a consent to a partner's ambiguous physical nonsexual actions. These ambiguous physical nonsexual actions included signals like moaning, eye contact, or a partner's increased heart rate that do not meet the standards of affirmative consent. While this study focused on college men and their reactions to affirmative consent policies on campus, the findings speak more broadly to narratives of consent-seeking and attribution. In the section to follow, I will discuss the implications of these empirical findings on research on men's sexual behaviors and the cultural scripts surrounding discussions of sexual violence more broadly.

It is extremely unlikely that every man in this study who attributed consent to a partner's ambiguous or mundane physical actions did so because they lacked compelling evidence that their previous sexual interactions were consensual. More likely, the participants in this study looked to ambiguous signals as a way to demonstrate their sexual skill and maintain power in their sexual encounters. Ambiguity in sexual narratives allows men to exacerbate their sexual prowess and, accordingly, demonstrate their masculinities (Currier 2013). In this case, the participants' narratives hint that their partners were eager to engage in sexual activity with them from the moment of first eye contact. Still, whether or not the men in this study committed acts of sexual violence is not all that is at stake in their varied applications of consent-seeking 
techniques. Equally as important are the narratives they endorse and normalize by relying on ambiguous signals to tell the stories of their sexual exploits. By sexualizing their partners' ambiguous and mundane actions, the men in this study make it more difficult to tell apart stories of consensual sex and sexual violence. Previous studies have found that sexual assailants use similar ambiguities to deny that they have committed acts of sexual assault and resist accountability (Scully and Marolla 1983). Furthermore, men's widespread willingness to accept mundane interactions like eye contact, moaning, or holding a conversation as evidence of consent upholds rape myths and creates a social context supportive of victim blaming (Hlavka 2014; Peterson and Muehlenhard 2004; Smith and Martinez 1996).

Relatedly, just as the participants' use of ambiguous sexual signals does not condemn them, it cannot absolve them either-even if nearly all of them endorsed affirmative consent. The men in this study only spoke highly of affirmative consent practices when asked explicitly about "affirmative consent." When we discussed their previous sexual experiences using more neutral words about their partners' frames of mind such as "willing," the participants' narratives included few references to consent-seeking behaviors that fit the affirmative consent model. These findings are consistent with Pascoe and Hollander's (2016) work, in which they argued that the label of "rapist" is stigmatizing to young men, indicating their failures of performing masculinity. Accordingly, men might tease each other with allegations of sexual assault as a way to disparage one another's masculinity and bolster their own, regardless of whether or not the teasers also have relied upon violence to obtain sexual access to women. The participants' widespread endorsement of "affirmative consent" might be subject to similar social desirability biases, particularly now that discussions of consent have become so explicitly linked with sexual 
assault prevention. It is possible that some of the men in this study have overstated their commitment to sexual assault prevention and seeking exclusively consensual sex.

Accordingly, the findings from this study cast past academic research on college students' gendered norms and sexual practices in a new light. On the topic of sexual consent, Jozkowski and colleagues $(2013 ; 2014)$ found that college men more commonly rely on physical cues to indicate consent than college women. The current study corroborates college men's dependence on physical cues and offers more detail about what young men consider to be physical signals of consent, which often have an ambiguous connection to sexual activity. Furthermore, the current study suggests that men may overstate their self-reported commitment to affirmative consent, especially in response to questions that use the word "consent" explicitly. As such, college men's use of verbal cues to indicate consent, as well as their commitment to sexual violence prevention may have been overstated in previous studies.

The current study also offers insight into gendered differences in interpretations of sexual initiation. In their work on college hookups, England and colleagues (2007) found that heterosexual men were more likely to attribute sexual initiation to women than the heterosexual women in the study. The findings from the current study suggest that some college men interpret women's mundane actions such as continuing a conversation as consent to- - and perhaps initiation of - a sexual encounter. In these scenarios, it is possible that men are the only ones who consider an interaction sexualized, allowing them greater control over sexual activity that may occur, despite attributing sexual initiation to their partners. These findings have practical implications for those adjudicating sexual assault complaints, as those accused commonly claim the event in question was consensual or even initiated by the complainant. The data from this study exemplify how claims of initiation may be contested and connected to a gendered 
hierarchy. Expanding administrators' understandings of gender dynamics in consent negotiations is crucial, particularly as many campus policymakers struggle to understand the nuances of consent themselves, inadvertently encouraging students to draw upon pre-existing and powerladen cultural norms (Degiuli and Nowotny 2019).

There are limitations to this study. While interviews are useful in understanding cultural narratives, the findings from this study rely heavily on self-reported data, which is subject to bias and limits analysis to the narratives crafted. While directly observing adolescents' sexual interactions would also certainly affect the data gathered by allowing the sorting of data of participants through their actual behaviors, there are other ways to bring multiple perspectives to the study of sexual signals. Matched pair interviews would be a useful step forward in triangulating the signals used by consenting sexual partners since both partners' accounts would be included in the data. Longitudinal research would be illuminating as well in attempts to better understand the effect of individual affirmative consent policies. Future research may also focus on asking more explicitly about sexual events that did not end in vaginal intercourse or sexual events that the participants opted to stop out of concern that their partner was not enthusiastically consenting. It is possible that men draw upon different culture scripts that are more similar to those espoused by affirmative consent policies when facing a potential partner's clear disinterest. Additionally, future research about the role of race in men's use of cultural scripts about sexual violence is needed as scripts about sexual violence perpetration—and particularly criminalization—are racialized.

In sum, the findings from this study indicate that recent attempts to educate men about affirmative consent and their responsibility to prevent sexual violence have likely reached them, but may not have led them to change their sexual behaviors or the way they invoke ambiguous 
signaling in narratives of their sexual encounters. Even when young men condone affirmative consent and claim to apply its teachings, they still rely on ambiguous and nonsexual physical cues as evidence that their partners consent to sexual activity. It is the use of these cues that reproduce the cultural notion that consent is unclear. 


\section{References}

Abbey, Antonia. 1982. 'Sex Differences for Friendly Behavior: Do Males Misperceive Females' Friendliness?” Journal of Personality and Social Psychology 42(5): 830-838.

Abbey, Antonia. 1987. "Misperceptions of Friendly Behavior as Sexual Interest: A Survey of Naturally Occurring Incidents.” Psychology of Women Quarterly 11: 173-194.

Armstrong, Elizabeth A., Laura Hamilton, and Brian Sweeney. 2008. "Sexual Assault on Campus: A Multilevel, Integrative Approach to Party Rape.” Social Problems 53(4): 483499.

Armstrong, Elizabeth A., Paula England, and Alison Fogarty. 2012. “Accounting for Women's Orgasm and Sexual Enjoyment in College Hookups and Relationships." American Sociological Review 77(3): 435-462.

Armstrong, Elizabeth A., Sandra Levitsky, Kamaria Porter, Miriam Gleckman-Krut, and Elizabeth Chase. 2019. "Defining Consent on Campus: What's in the Media vs. What's in the Policies." Council on Contemporary Families Symposium: Defining Consent.

Badke, Lara K., Kamaria B. Porter, Jessica R. Garrick, Elizabeth A. Armstrong, and Sandra L. Levtisky. 2016. “Compliance or Rhetoric: Are Campus Sexual Misconduct Policies Fulfilling Title IX Legal Obligations?” Columbus, OH: Association for the Study of Higher Education Annual Conference.

Beres, Melanie A. 2010. "Sexual Miscommunication? Untangling Assumptions about Sexual Communication between Casual Sex Partners." Culture, Health, and Sexuality 12(1): 114.

Bridges, Tristan. 2010. "Men Just Weren't Made To Do This: Performances of Drag at 'Walk a Mile in Her Shoes' Marches." Gender \& Society 24(1): 5-30. 
Bridges, Tristan, and CJ Pascoe. 2014. "Hybrid Masculinities: New Directions in the Sociology of Men and Masculinities.” Sociology Compass 8(3): 246-258.

California State Legislature. 2014. State Senate. Student Safety: Sexual Assault S.B. 967.

Cantor, David, Bonnie Fisher, Susan Chibnall, Resanne Townsend, Hyunshik Lee, Carol Bruce, and Gail Thomas. 2015. "Report on the AAU Campus Climate Survey on Sexual Assault and Sexual Misconduct." The Association of American Universities.

Connell, R.W., and James W. Messerschmidt. 2005. "Hegemonic Masculinity: Rethinking the Concept.' Gender \& Society 19(6): 829-859.

Currier, Danielle. 2013. "Strategic Ambiguity: Emphasized Femininity and Hegemonic Masculinity in the Hookup Culture.” Gender \& Society 27(5): 704-727.

Degiuli, Francesca and Jordan Nowotny. 2019. "The Administration of Consent: An Exploration of How Consent Education is Understood and Implemented at a Small Private University." Sexuality \& Culture. Online first.

England, Paula, Emily Fitzgerald Shafer, and Alison C.K. Fogarty. 2007. "Hooking Up and Forming Romantic Relationships on Today’s College Campuses”. Pp. 531-547 in The Gendered Society Reader, Third Edition, edited by Michael Kimmel and Amy Aronson. New York, NY: Oxford University Press.

Frese, Bettina, Moya, M.C. Gandía, and Jesús L. Megías. 2004. "Social Perception of Rape: How Rape Myth Acceptance Modulates the Influence of Situational Factors." Journal of Interpersonal Violence 19(2): 143-161.

Friedman, Jaclyn, and Jessica Valenti. 2008. Yes Means Yes! Visions of Female Sexual Power and a World Without Rape. Berkeley, CA: Seal Press. 
Hickman, Susan E., and Charlene L. Muehlenhard. 1999. “'By the Semi-mystical Appearance of a Condom': How Young Women and Men Communicate Sexual Consent in Heterosexual Situations.” The Journal of Sex Research 36(3): 258-272.

Hlavka, Heather. 2014. "Normalizing Sexual Violence: Young Women Account for Harassment and Abuse." Gender \& Society 28(3): 337-358.

Hirsch, Jennifer and Shamus Khan. 2020. Sexual Citizens: A Landmark Study on Sex, Power, and Assault on Campus. New York, NY: W.W. Norton \& Company.

Hirsch, Jennifer S., Shamus R. Khan, Alexander Wamboldt, and Claude E. Mellins. 2019. “Social Dimensions of Sexual Consent Among Cisgender Heterosexual College Students: Insights From Ethnographic Research.” Journal of Adolescent Health 64(1): 26-35.

Holland, Kathryn J., and Cortina Lillia, M. 2017. “It Happens to Girls All the Time'; Explaining Sexual Assault Survivors' Reasons For Not Using Campus Supports.” Journal of Community Psychology 59(1): 50-64.

Jacques-Tiura, Angela J., Antonia Abbey, Michele R. Parkhill, and Tina Zawacki. 2007. "Why Do Some Men Misperceive Women's Sexual Intentions More Frequently Than Others Do? An Application of the Confluence Model.” Personality and Social Psychology Bulletin 33(11): 1467-1480.

Jozkowski, Kristin N., and Zoe D. Peterson. 2013. “College Students and Sexual Consent: Unique Insights.” Journal of Sex Research 50(6): 517-523.

Jozkowski, Kristin N., Zoe D. Peterson, Stephanie A. Sanders, Barbara Dennis, and Michael Reece. 2014. “Gender Differences in Heterosexual College Students' Conceptualizations and Indicators of Sexual Consent: Implications for Contemporary Sexual Assault Prevention Education.” Journal of Sex Research 51(8) 904-916. 
King, Bruce M., Megan R. Fallon, Elizabeth R. Reynolds, Kennedy L. Williamson, Anyah Barber, and Angela R. Giovinazzo. 2020. Journal of Interpersonal Violence. Online first. Kitzinger, Celia, and Hannah Frith. 1999. "Just Say No? The Use of Conversation Analysis in Developing a Feminist Perspective on Sexual Refusal.” Discourse \& Society 10(3): 293316.

Krebs, Christopher P., Christine H. Lindquist, Tara D. Warner, Bonnie S. Fisher, and Sandra L. Martin. 2007. “The Campus Sexual Assault (CSA) Study.” U.S. Department of Justice.

Levin, Roy J., and Willy van Berlo. 2004. “Sexual Arousal and Orgasm in Subjects Who Experience Forced or Non-consensual Sexual Stimulation - A Review." Forensic and Legal Medicine 11(2): 82-88.

Malamuth, Neil M., Mark Huppin, and Daniel Linz. 2018. "Sexual Assault Interventions May be Doing More Harm Than Good With High-risk Males." Aggression and Violent Behavior 41: $20-24$.

Martin, Patricia Yancey. 2016. "The Rape Prone Culture of Academic Contexts: Fraternities and Athletics." Gender \& Society 30(1): 30-43.

Masters, N. Tatiana. 2010. “My Strength is Not For Hurting': Men's Anti-rape Websites and the Construction of Masculinity and Male Sexuality." Sexualities 13(1): 33-46.

Messner, Michael. 2016. "Bad Men, Good Men, Bystanders: Who is the Rapist?" Gender \& Society 30(1): 57-66.

Muehlenhard, Charlene L., Terry P. Humphreyes, Kristn N. Jozokowski, and Zoë D. Peterson. 2016. "The Complexities of Sexual Consent among College Students: A Conceptual and Empirical Review." The Journal of Sex Research 53(4/5): 457-487.

New York State Senate. 2015. S.B. S5965. 
Osman, Suzanne L. 2006. 'Predicting Men's Rape Perceptions Based on the Belief That 'No' Really Means 'Yes.'” Journal of Applied Social Psychology 33(4): 683-692.

Pascoe, C.J., and Jocelyn A. Hollander. 2016. “Good Guys Don’t Rape: Gender, Domination, and Mobilizing Rape.” Gender \& Society 30(1): 67-79.

Peterson, Zoë, and Charlene L. Muehlenhard. 2004. “Was it Rape? The Function of Women's Rape Myth Acceptance and Definitions of Sex in Labeling Their Own Experiences.” Sex Roles 51(3/4): 129-144.

Pugh, Brandie, Holly Ningard, Thomas Vander Ven, and Leah Butler. 2016. "Victim Ambiguity: Bystander Intervention and Sexual Assault in the College Drinking Scene." Deviant Behavior 37(4): 401-418.

Randall, Melanie. 2010. “Sexual Assault Law, Credibility, and 'Ideal Victims': Consent, Resistance, and Victim Blaming." Canadian Journal of Women and the Law 22(2): 397433.

Ryan, William. 1976. Blaming the victim. Vintage.

Scully, Diana, and Joseph Marolla. 1983. “Convicted Rapists’ Vocabulary of Motive: Excuses and Justifications.” Social Problems 31(5): 530-544.

Simon, William, and John H. Gagnon. 1986. "Sexual Scripts: Permanence and Change." Archives of Sexual Behavior 15(2): 97-120.

Smith, Andrew R. and Jacqueline M. Martinez. 1995. "Signifying Harassment: Communication, Ambiguity and Power." Human Studies 18: 63-87.

Stepp, Laura Sessions. 2007. “A New Kind of Date Rape.” Cosmopolitan, September 11. 
Swartout, Kevin M., Mary P. Koss, Jacquelyn W. White, Martie P. Thompson, Antonia Abbey, and Alexandra L. Bellis. 2015. "Trajectory Analysis of Campus Serial Rape Assumption.” JAMA Pediatrics 169(12): 1148-1154.

Wade, Lisa. 2017. American Hookup: The New Culture of Sex on Campus. New York, NY: W.W. Norton \& Company.

Warshaw, Robin. 1988. I Never Called It Rape: The Ms. Report on Recognizing, Fighting, and Surviving Date Rape. New York, NY: HarperCollins Books. 
Appendix A: Figures and Tables

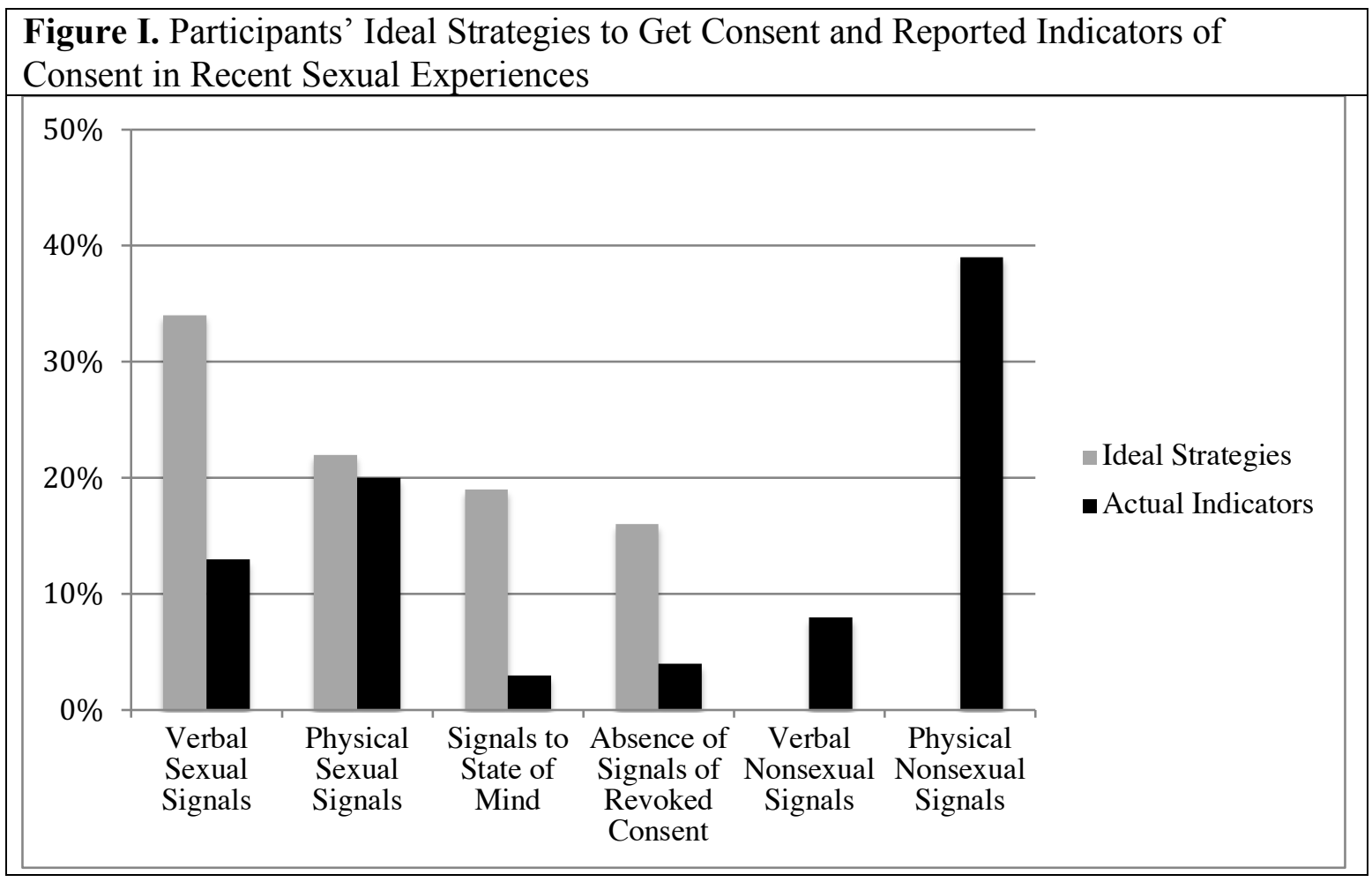

Table I. Common Physical Nonsexual Signals of Sexual Consent

\begin{tabular}{|c|c|}
\hline Signal & Frequency \\
\hline Moaning & 25 \\
\hline Making eye contact & 11 \\
\hline Touching me & 8 \\
\hline Elevated breath rate & 8 \\
\hline Grabbing me & 8 \\
\hline Scratching me & 7 \\
\hline Talking to me & 6 \\
\hline Getting onto a bed & 5 \\
\hline Making faces & 5 \\
\hline Elevated heart rate & 4 \\
\hline Bringing me drinks & 3 \\
\hline Dancing together & 3 \\
\hline Drinking alcohol & 3 \\
\hline
\end{tabular}

